Irradiation Effects on RIA

Fragmentation CU Beam Dump

S. Reyes, J. L. Boles, L. E. Ahle, W. Stein, B. D. Wirth

May 11, 2005

Particle Accelerator Conference 2005

Knoxville, TN, United States

May 16, 2005 through May 20, 2005 
This document was prepared as an account of work sponsored by an agency of the United States Government. Neither the United States Government nor the University of California nor any of their employees, makes any warranty, express or implied, or assumes any legal liability or responsibility for the accuracy, completeness, or usefulness of any information, apparatus, product, or process disclosed, or represents that its use would not infringe privately owned rights. Reference herein to any specific commercial product, process, or service by trade name, trademark, manufacturer, or otherwise, does not necessarily constitute or imply its endorsement, recommendation, or favoring by the United States Government or the University of California. The views and opinions of authors expressed herein do not necessarily state or reflect those of the United States Government or the University of California, and shall not be used for advertising or product endorsement purposes. 


\title{
IRRADIATION EFFECTS ON RIA FRAGMENTATION CU BEAM DUMP*
}

\author{
S. Reyes ${ }^{\#}$, J. L. Boles, L. E. Ahle, W. Stein, LLNL, Livermore, CA 94550, U.S.A. \\ B. D. Wirth, UCB, Berkeley, CA 94720-1730, U.S.A.
}

Abstract

Within the scope of conceptual research and development (R\&D) activities in support of the Rare Isotope Accelerator (RIA) facility, high priority is given to the development of high-power fragmentation beam dumps. A pre-study was made of a static water-cooled $\mathrm{Cu}$ beam dump that can meet requirements for a $400 \mathrm{MeV} / \mathrm{u}$ uranium beam. The issue of beam sputtering was addressed and found to be insignificant. Preliminary radiation transport simulations show significant damage (in displacements per atom, DPA) in the vicinity of the Bragg peak of the uranium ions. Experimental data show that defects in $\mathrm{Cu}$ following neutron or high-energy particle irradiation tend to saturate at doses between 1 and 5 DPA, and this saturation in defect density also results in saturation of mechanical property degradation. However, effects of swift heavy ion irradiation and the production of gaseous and solid transmutant elements still need to be addressed. Initial calculations indicate that $\mathrm{He}$ concentrations on the order of 400 appm are produced in the beam dump after several weeks of continuous operation and $\mathrm{He}$ embrittlement may be a concern. Recommendations are made for further investigation of $\mathrm{Cu}$ irradiation effects for RIA-relevant conditions.

\section{BEAM DUMP DESCRIPTION}

The RIA project [1] is a new US Department of Energy (DOE) accelerator facility initiative currently at the preconceptual design stage. The mission of the RIA project is to provide a world-class facility for performing cuttingedge research on nuclear structure, nuclear astrophysics, and fundamental interactions and symmetries.

RIA will have one or more fragmentation stations that will accept up to $400 \mathrm{~kW}$ heavy ion beams. One of the most difficult technical challenges may be the collection of the un-reacted primary beam and unwanted fragments in the early "pre-separator" stages of the fragment separator beam lines. Approximately 20 to $30 \%$ of the $400 \mathrm{~kW}$ of primary beam will be deposited in the production target. The remainder will continue into the first dipole of the separator where it will be spatially separated from the fragment of interest, and finally will impact onto a beam dump located downstream from the first dipole.

Designing the fragmentation beam dumps for RIA represents a critical challenge. A design requirement is to make an adaptable beam dump system that intercepts unwanted fragments and the non-reacting primary beam, yet does not cut into the phase space of the desired fragments. Therefore, the dump may have to cover a wide area or be movable, and is required to absorb up to 400

\footnotetext{
*Work performed under the auspices of the U. S. Department of Energy by University of California Lawrence Livermore National Laboratory under Contract W-7405-Eng-48.

"reyes20@1lnl.gov
}

$\mathrm{kW}$ (in the case of target malfunction) at extremely high power density. Also, the system should be compact to avoid optical aberrations in the system, and mechanically simple to facilitate remote handling. In addition to extreme thermal loadings, the system must survive the radiation damage induced by the beam stopping in material.

Over the past two years, neutronics personnel at Lawrence Livermore National Laboratory (LLNL) have collaborated with experts at Michigan State University (MSU), Oak Ridge National Laboratory (ORNL) and Argonne National Laboratory (ANL), for RIA R\&D activities regarding the calculations of radiation fields, shielding and material damage in the fragmentation target station. Led by LLNL, a static wedge-shaped beam dump concept has been developed that can meet requirements for the available beam powers and power densities for a $320 \mathrm{MeV} / \mathrm{A}$ uranium beam. A schematic is shown in Figure 1. The beam dump is located downstream of the dipole magnet and consists of two $1-\mathrm{cm}$ thick watercooled $\mathrm{Cu}$ plates in a $5^{\circ}$ angle. A beam spot size of $5 \times 1$ $\mathrm{cm}^{2}$ is assumed at the entrance of the dump.

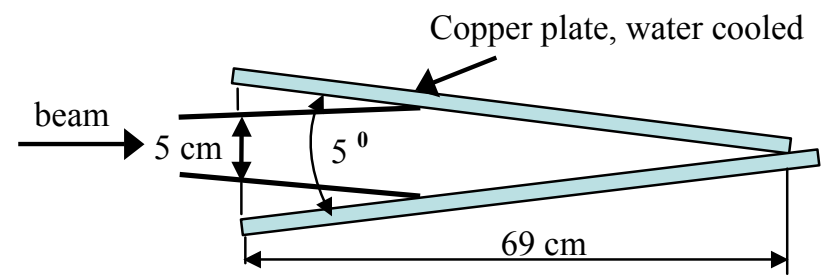

Figure 1. Side view of water-cooled $\mathrm{Cu}$ wedge beam dump concept for RIA fragmentation target area

\section{IRRADIATION EFFECTS}

As mentioned in the previous section, there are a number of design requirements for the RIA fragmentation beam dump. While it is feasible to make reliable estimates and assessments regarding mechanical design and thermodynamic performance of the dump, this is not the case for the issue of radiation damage induced from the high power heavy-ion beam. This paper will describe the analyses performed to address the sputtering and radiation damage of the $\mathrm{Cu}$ wedge beam dump concept due to RIA operation with a $320 \mathrm{MeV} / \mathrm{A}$ uranium beam.

\section{Sputtering results}

We have used the SRIM code [2] to simulate sputtering effects on the $\mathrm{Cu}$ beam dump by the energetic $\mathrm{U}$ ions. When modeling the irradiation of $\mathrm{Cu}$ by ions at large energies, we found massive cascades that exceed the code's limitations (>8000 atoms per cascade). In SRIM, this limitation can be overcome by modeling just a thin layer of material in the direction normal to the surface. This limits the number of collisions to be tracked, as only 
the events close to the material's surface will contribute to sputtering. For a $87.5^{\circ}$ incidence angle, however, a few angstroms in direction normal to the surface still involve a long distance in the direction of the beam and therefore, a large number of collisions are tracked as part of the calculation, making the code slow and unstable. After performing a parametric assessment for various ion energies at $87.5^{\circ}$, we determined that simulations were non practical for $>100 \mathrm{MeV} /$ nucleon.

In order to determine the effect of the beam energy on the sputtering yield we simulated a simple case with the surface at normal incidence. At $0^{\circ}$ incidence, we found that the yield reaches a peak for a particular ion energy and later decreases for increasing ion energy (Figure 2). This indicates that if we can determine what the maximum sputtering yield is for the $87.5^{\circ}$ case, we could use that value as an upper bound for the higher energy cases relevant for RIA. At $0^{\circ}$, the maximum sputtering yield was found for $0.5 \mathrm{MeV} /$ ion, which corresponds to an ion range of $457 \mathrm{~A}$. For the case of $87.5^{\circ}$ incidence angle, an energy of $13 \mathrm{MeV} /$ ion with corresponding range of 10500 A $(\sim 457 / \cos (87.5))$, would result in equivalent penetration depth in the direction normal to the surface. The SRIM results shown in Figure 2 confirm that the maximum yield at $87.5^{\circ}$ happens for ion energy of 13 $\mathrm{MeV}$, then decreases for longer ion ranges. Assuming a current of $3 \times 10^{13}$ particles per second (pps) during several months of continuous operation, the estimated surface material removal will be $<1 \mathrm{~mm}$, even at the maximum yield of $\sim 300$ at/ion. Therefore, it is expected that sputtering will not be an issue for the RIA beam dump.

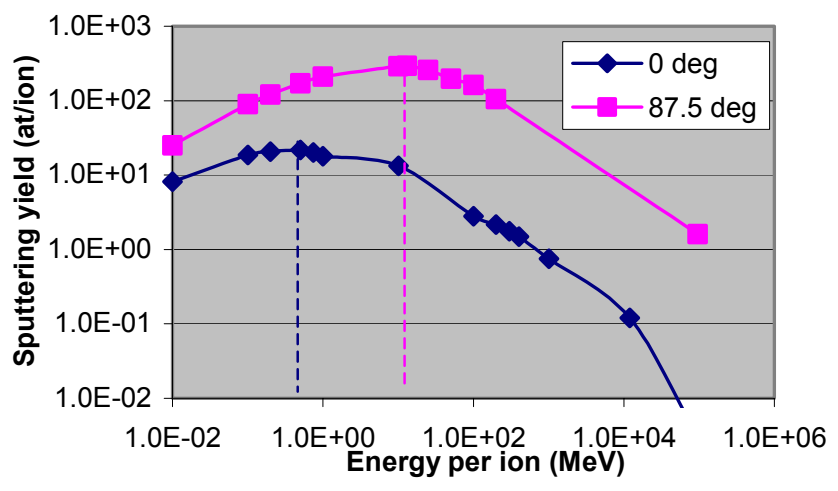

Figure 2. Sputtering yield as a function of ion energy

\section{DPA results}

As part of the RIA neutronics collaboration, we have established a set of state-of-the-art of computer codes and libraries for ion transport calculations, including analysis of particle fluxes, isotope yield, nuclear heating and material damage for the components of interest. Some of the codes currently included in our methodology are MCNPX [3], MARS15 [4], and PHITS [5]. Presently, PHITS is the only readily available, 3-D code capable of transporting high-energy heavy ions. Both MCNPX and MARS are close to releasing updated versions that include heavy ion transport, but have not done so to date.
Therefore, we have used the code PHITS to simulate the transport of the heavy ions in the RIA fragmentation station. In particular, we have created a model of the wedge-shaped $\mathrm{Cu}$ beam dump in order to study the ion transport and the radiation damage in terms of DPA. In this model, we have subdivided each $\mathrm{Cu}$ plate into thin regions in order to properly track the particles and identify the maximum DPA value, which occurs around the Bragg peak for the stopping uranium ions $(\sim 3 \mathrm{~mm}$ for 320 $\mathrm{MeV} / \mathrm{A}$ ions). The results for the DPA from the primary beam, neutrons and protons are shown in Figures 3 and 4, for two different region (or mesh) thicknesses.

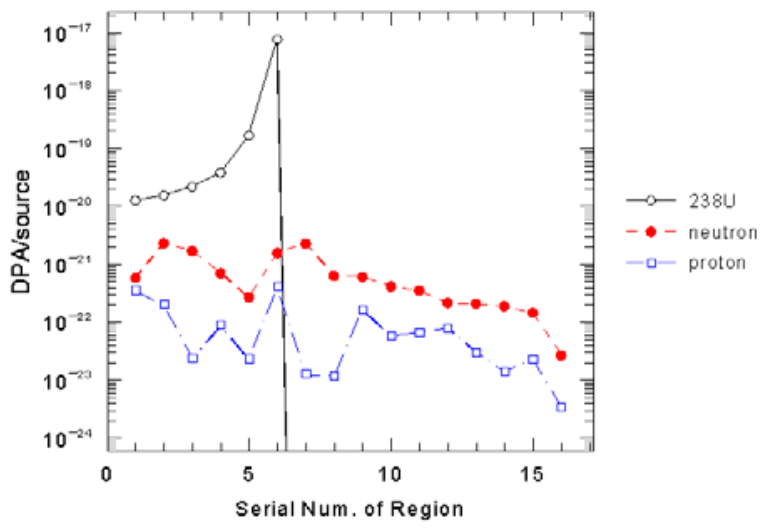

Figure 3. DPA from $320 \mathrm{MeV} / \mathrm{A} \mathrm{U}$ ions on $\mathrm{Cu}$ beam dump as a function of depth, case of $25 \mu \mathrm{m}$ regions.

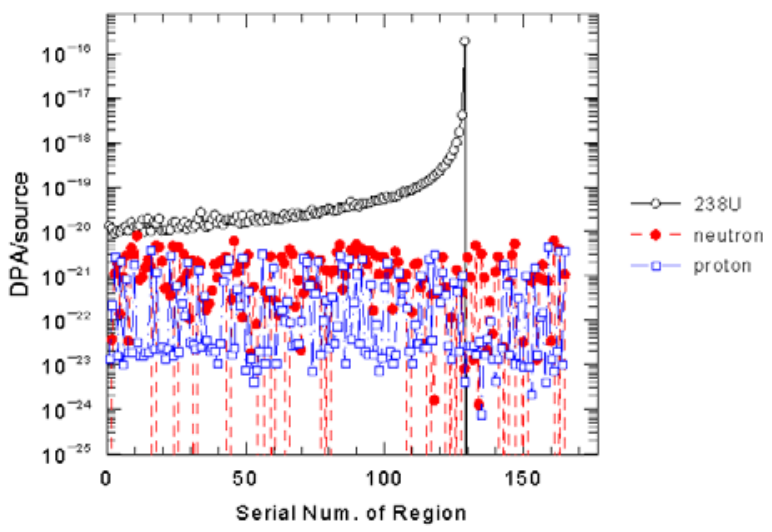

Figure 4. DPA from $320 \mathrm{MeV} / \mathrm{A} \mathrm{U}$ ions on $\mathrm{Cu}$ beam dump as a function of depth, case of $1 \mu \mathrm{m}$ regions.

In both of these plots the DPA peak occurs at a depth of $\sim 130 \mu \mathrm{m}(3 \mathrm{~mm} * \cos (87.5))$. The plots also indicate that the thickness of the mesh is relevant for evaluation of the maximum DPA, and that a compromise has to be reached between efficient modeling and reasonable computing time. It must be noted that although the results shown in these plots maybe underestimated, some important conclusions can be drawn from here. Figure 3 shows peak damage rates as high as $\sim 25$ DPA per day for full power operations, assuming a current of $3 \times 10^{13}$ pps. Many past applications have used a few DPA as the limit of acceptable radiation damage [6]. If those same limits apply here, then our result would imply an unacceptably short lifetime for the fragment separator beam dump. 
However, there is still some uncertainty as to whether the predictions from PHITS are accurate. While some benchmarking of this code in heavy ion transport and DPA calculations for neutrons and protons has occurred, the DPA calculations for heavy ions have not been validated due to the lack of experimental data.

Previous data from neutron and high energy proton irradiation of copper have shown irradiation embrittlement, which tends to saturate at doses between 1 and 5 DPA. Thermal conductivity decreases are also believed to saturate at a level of about $10-20 \%$ decrease [7]. However, it is known that for heavy ions above about $100 \mathrm{MeV}$, the electronic energy losses become much larger and can lead to dramatic effects on materials. Very little data exists about heavy ion irradiation of metals, and it is recommended that fundamental research investigate swift heavy ion effects, as well as damage saturation from high energy uranium ions on copper.

\section{Gas production}

The individual effects of helium and hydrogen implantation on structural materials are relatively well established [8-10]. Large quantities of helium in metals can increase the strength, decrease the ductility, reduce the creep and stress rupture properties, decrease the fatigue life and weldability, and promote swelling. Hydrogen can also adversely affect the mechanical properties and promote premature fracture along metallurgical interfaces. Furthermore, the adverse effects of hydrogen and helium implantation may couple with displacement damage to limit component lifetime in a system. This evidence illustrates the potential importance of these interactions to the safe and efficient utilization of materials in intense, high-energy radiation environments such as RIA. Gas production in the beam dump $\mathrm{Cu}$ plates could lead to degradation of the mechanical properties of the material.

Using results of residual nucleii yield from our PHITS model for the wedge-shaped beam dump we have estimated concentration of $\mathrm{H}$ and $\mathrm{He}$ gas in the copper. We have obtained $\sim 400$ appm He and $\sim 1200$ appm of $\mathrm{H}$ in the beam dump after 2 months of continuous operation. These levels of gas production can be a concern for hardening/embrittlement effects in the beam dump and investigation of these issues, including experiments, are strongly recommended.

\section{CONCLUSIONS}

A static wedge-shaped beam dump concept has been proposed for the RIA fragmentation line, consisting of two water-cooled $\mathrm{Cu}$ plates at a $5^{\circ}$ angle. Such concept meets the requirements for the available beam powers and power densities at RIA, however potential degradation of mechanical properties due to radiation damage is a concern.
In this work we have addressed several issues related to irradiation effects on this beam dump concept assuming operation with a $320 \mathrm{MeV} / \mathrm{A}$ uranium beam. First, the issue of beam sputtering was addressed and shown not to be significant.

We have simulated the uranium transport into the beam dump using the 3D heavy ion transport code PHITS. Results show significant displacements in the vicinity of the Bragg peak of uranium ions. Although experimental data from particle irradiation show that defects in $\mathrm{Cu}$ tend to saturate at doses of a few DPA, effects of swift heavy ion irradiation may lead to a different material response and therefore need to be addressed.

Finally, we have estimated the production of $\mathrm{H}$ and $\mathrm{He}$ gas within the $\mathrm{Cu}$ beam dump. Results from PHITS simulations show levels of a several hundred appm for $\mathrm{H}$ and He, which are likely to adversely affect the mechanical properties of the beam dump. Moreover, the effects of gas transmutants may couple with displacement damage to limit the beam dump lifetime.

Investigation of $\mathrm{Cu}$ and other potential candidates performance under energetic heavy ions at currently available facilities is strongly encouraged.

\section{REFERENCES}

[1] See www.orau.org./ria/.

[2] J. F. Ziegler, see www.SRIM.org.

[3] H. Iwase, et al., "Development of heavy ion transport Monte Carlo code", Nucl. Inst. Meth. Phys. Res. B183, p. 374 (2001).

[4] H.G. Hughes, et al., "MCNPX for Neutron-Proton Transport”, International Conference on Mathematics \& Computation, Reactor Physics \& Environmental Analysis in Nuclear Applications, ANS, Madrid, Spain, September 27-30, 1999.

[5] N.V. Mokhov, et al., "Recent Enhancements to the MARS15 Code", Fermilab-Conf-04/053 (2004); http://www-ap.fnal.gov/MARS/.

[6] M.H. Barnett, et al., "Radiation damage to the 316 stainless steel target container vessel at SNS," $J$. Nucl. Mat., 296, p. 54 (2001).

[7] S.A. Fabritsiev, et al., "Low-temperature radiation embrittlement of copper alloys," J. Nucl. Mat., 233237, p. 513 (1996).

[8] H. Ullmaier, "The Influence of Helium on the Bulk Properties of Fusion Reactor Structural Materials," Nuclear Fusion, 24, p. 1039, (1984).

[9] J. Morisawa, et al., "Effects of Hydrogen on Mechanical Properties in Irradiated Austenitic Stainless Steels," J. Nucl. Mat., 212-215, p. 1396, (1994).

[10]E. Abramov, et al., "Surface Behavior of First-Wall Materials due to the Synergistic Effect of Helium and Hydrogen Isotopes," J. Nucl. Mat., 212-215, p. 1390, (1994). 